\title{
Regular coverings of the integers by arithmetic progressions
}

by

R. J. Simpson (Adelaide, Australia)

1. Introduction. We write $\langle a, d\rangle$ for the arithmetic progression, henceforth AP, consisting of all integers congruent to $a$ modulo $d$.

Definition 1. A collection $\mathscr{A}=\left\{\left\langle a_{i}, d_{i}\right\rangle: i=1, \ldots, t\right\}$ of AP's is called a regular covering if every integer belongs to at least one AP in $\mathscr{A}$, and no subcollection has this property. We write $\mid \mathscr{A}$ for the number of AP's in $\mathscr{A}$.

DEFINITION 2. A disjoint covering is a regular covering in which each integer belongs to exactly one AP.

We will also need the following function. If the canonical prime factorization of $n$ is

$$
n=\prod_{i=1}^{t} p_{i}^{\alpha_{i}}
$$

then

$$
f(n)=\sum_{i=1}^{t} \alpha_{i}\left(p_{i}-1\right)
$$

We note that $f(n)$ is a completely additive function.

In [4] $\breve{S}$. Znám conjectured that if $\mathscr{A}$ is a disjoint covering and $P$ is the least common multiple of the moduli of the AP's in $\mathscr{A}$, then

$$
|\mathscr{A}| \geqslant f(P)+1 \text {. }
$$

In [2] and [3] Znám proved some results in this direction and in [1] Korec proved the conjecture.

In [5] Znám conjectured that $\mathscr{A}$ need only be regular for (1.1) to hold. In Theorem 2 of the present paper we prove a result slightly stronger than this, the conjecture itself being proved in the first corollary to that theorem.

In Section 2 we prove some straightforward lemmata and in Section 3 we prove Theorem 1 which gives a general property of regular coverings. The corollary to this theorem is used in the proof of Theorem 2 . 


\section{Some lemmata.}

LEMMA 1. (i) $\langle a, d\rangle$ intersects $\langle A, D\rangle$ if and only if

$$
a \equiv A(\bmod (d, D))
$$

where $(d, D)$ is the greatest common divisor of $d$ and $D$.

(ii) If $\langle a, d\rangle$ intersects both $\left\langle A_{1}, D_{1}\right\rangle$ and $\left\langle A_{2}, D_{2}\right\rangle$ then

$$
A_{1} \equiv A_{2}\left(\bmod \left(d, D_{1}, D_{2}\right)\right) \text {. }
$$

Proof. (i) The intersection of $\langle a, d\rangle$ and $\langle A, D\rangle$ consists of those integers $x$ satisfying

$$
x \equiv a(\bmod d), \quad x \equiv A(\bmod D) .
$$

By the Chinese Remainder Theorem these congruences are simultaneously solvable if and only if $a \equiv A(\bmod (d, D))$.

(ii) By (i) we have

$$
a \equiv A_{1}\left(\bmod \left(d, D_{1}\right)\right) \quad \text { and } \quad a \equiv A_{2}\left(\bmod \left(d, D_{2}\right)\right)
$$

which imply the result.

Lemma 2. Suppose $\mathscr{A}=\left\{\left\langle a_{i}, d_{i}\right\rangle: i=1, \ldots, t\right\}$ is a regular covering and $p$ divides $d_{j}$ for some $j$ where $p$ is a prime. Then the set

$$
\left\{a_{i}: p\left\{d_{i}\right\}\right.
$$

contains a complete set of residues modulo $p$. Here $p \mid d_{i}$ means $p$ divides $d_{i}$.

Proof. Suppose there is a residue class $r \bmod p$ such that $\alpha$ contains no AP for which $p$ divides $d_{i}$ and $a_{i}$ is congruent to $r \bmod p$. Then the integers congruent to $r \bmod p$ must be covered by AP's in the collection

$$
\mathscr{B}=\left\{\left\langle a_{i}, d_{i}\right\rangle \in \mathscr{A}: p \nmid d\right\} .
$$

By the regularity of $\mathscr{A}$ there is some integer $x_{0}$ which is not an element of any AP in $\not B$. Let $P$ be the least common multiple of the moduli of AP's occurring in and choose $x$ so that

$$
x \equiv x_{0}(\bmod P) \quad \text { and } \quad x \equiv r(\bmod p) .
$$

Then $x$ does not belong to any AP in $\mathscr{A}$. This contradiction proves the lemma.

Lemma 3 (Reduction of a collection of AP's). (i) Suppose $\left\langle\left\langle a_{i}, d_{i}\right\rangle\right.$ : $i=1, \ldots, t\}$ is a minimal covering of the $A P\langle a, d\rangle$ and

$$
\delta_{i}=\left(d, d_{i}\right) \quad \text { for } \quad i=1, \ldots, t .
$$

If we construct another collection of AP's

$$
\mathscr{A}^{*}=\left\{\left\langle a_{i}^{*}, d_{i}^{*}\right\rangle: i=1, \ldots, t\right\}
$$

where

$$
d_{i}^{*}=d_{i} / \delta_{i} \quad \text { and } \quad a_{i}^{*} d / \delta_{i} \equiv\left(a_{i}-a\right) / \delta_{i}\left(\bmod d_{i}^{*}\right),
$$

then $\mathscr{S}^{*}$ is a regular covering.

(ii) In particular, if $d$ divides $d_{i}$

$$
d_{i}^{*}=d_{i} / d, \quad a_{i}^{*} \equiv \frac{a_{i}-a}{d}\left(\bmod d_{i}^{*}\right) .
$$

Proof. (i) Note that $\delta_{i}$ divides $a_{i}-a$ by (i) of Lemma 1 . Let $m$ be any integer. We claim that $m$ belongs to $\left\langle a_{i}^{*}, d_{i}^{*}\right\rangle$ if and only if $a+m d$ belongs to $\left\langle a_{i}, d_{i}\right\rangle$. For,

$$
\begin{aligned}
a+m d \in\left\langle a_{i}, d_{i}\right\rangle & \Leftrightarrow m d / \delta_{i} \equiv\left(a_{i}-a\right) / \delta_{i}\left(\bmod d_{i}^{*}\right) \\
& \Leftrightarrow m \in\left\langle a_{i}^{*}, d_{i}^{*}\right\rangle .
\end{aligned}
$$

Thus $\mathscr{A}^{*}$ covers the integers, and if any proper subset of $\mathscr{A}^{*}$ covers the integers then a proper subset of $\mathscr{A}$ would cover $\langle a, d\rangle$, contradicting the minimality of $\%$. Thus $\mathscr{A}^{*}$ is regular.

(ii) If $d$ divides $d_{i}$ then $\delta_{i}$ equals $d$ and the result follows.

When using the construction described in this lemma we will say $x^{*}$ is the reduction of a via $\langle a, d\rangle$.

\section{The first theorem.}

THEOREM 1, Suppose $\mathscr{A}$ is regular, $\langle a, d\rangle \in \mathscr{A}$ and $p^{\alpha}$ is the highest power of a prime $p$ which divides $d$. Then

(i) for $1 \leqslant k \leqslant \alpha, \propto$ has a subcollection,$\alpha_{k}$ where

$$
\mathscr{A} \mathscr{A}_{k}=\left\{\left\langle a_{i}^{(k)}, d_{i}^{(k)}\right\rangle: 1 \leqslant i \leqslant p-1\right\}
$$

such that for each $i$ satisfying $1 \leqslant i \leqslant p-1$,

$$
\begin{gathered}
p_{i}^{k} \mid d_{i}^{(k)}, \\
a_{i}^{(k)} \equiv a\left(\bmod p^{k-1}\right), \\
\frac{a_{i}^{(k)}-a}{p^{k-1}} \equiv i(\bmod p) .
\end{gathered}
$$

(ii) The $\alpha(p-1) A P^{\prime} s\left\langle a_{i}^{(k)}, d_{i}^{(k)}\right\rangle$ are pairwise disjoint, and each is disjoint from $\langle a, d\rangle$.

Proof. (i) We prove the result for an arbitrary value of $k$.

Let $\mathscr{G}$ be a minimal subcollection of $\&$ such that $\mathscr{C}$ covers $\left\langle a, p^{k-1}\right\rangle$ and let $\mathscr{C}^{*}$ be the reduction of $\mathscr{E}$ via $\left\langle a, p^{k-1}\right\rangle$. Now $\langle a, d\rangle$ is a subset of $\left\langle a, p^{k-1}\right\rangle$ so the regularity of $\mathscr{A}$ implies that $\langle a, d\rangle$ belongs to $\mathscr{C}$. By (ii) of Lemma $3\left\langle 0, d / p^{k-1}\right\rangle$ belongs to $\mathscr{C}^{*}$. Since $p$ divides $d / p^{k-1}$ Lemma 2 implies that $\mathscr{C}^{*}$ contains a further $p-1$ AP's $\left\langle a_{1}^{*}, d_{1}^{*}\right\rangle, \ldots,\left\langle a_{p-1}^{*}, d_{p-1}^{*}\right\rangle$ such that 
$\left\{0, a_{1}^{*}, \ldots, a_{p-1}^{*}\right\}$ is a complete residue system modulo $p$ and that each $d_{i}^{*}$ is divisible by $p$.

For each $i$, let $\left\langle a_{i}^{(k)}, d_{i}^{(k)}\right\rangle$ be the $\mathrm{AP}$ of which $\left\langle a_{i}^{*}, d_{i}^{*}\right\rangle$ is the reduction. Then by Lemma 3,

$$
d_{i}^{*}=\frac{d_{i}^{(k)}}{\left(p^{k-1}, d_{i}^{(k)}\right)} .
$$

Since $p$ divides $d_{i}^{*}$ this implies that $p^{k}$ divides $d_{i}^{(k)}$. Now $\left\langle a_{i}^{(k)}, d_{i}^{(k)}\right\rangle$ intersects $\left\langle a, p^{k-1}\right\rangle$ so by (i) of Lemma 1 ,

$$
a_{i}^{(k)} \equiv a\left(\bmod p^{k-1}\right)
$$

and by (ii) of Lemma 3 ,

$$
a_{i}^{*} \equiv \frac{a_{i}^{(k)}-a}{p^{k-1}}(\bmod p)
$$

Since $a_{i}^{*}$ runs through a reduced residue system modulo $p$ we can, by appropriate ordering, ensure that,

$$
\frac{a_{i}^{(k)}-a}{p^{k-1}} \equiv i(\bmod p)
$$

(ii) We prove this part by contradiction. Suppose $\left\langle a_{i}^{(k)}, d_{i}^{(k)}\right\rangle$ intersects $\left\langle a_{i^{\prime}}^{\left(k^{\prime}\right)}, d_{i^{\prime}}^{\left(k^{\prime}\right)}\right\rangle$ where $k^{\prime} \geqslant k$ so that $p^{k}$ divides $\left(d_{i}^{(k)}, d_{i^{\prime}}^{\left(k^{\prime}\right)}\right)$. Then by (i) of Lemma 1 ,

$$
a_{i}^{(k)} \equiv a_{i^{\prime}}^{\left(k^{\prime}\right)}\left(\bmod p^{k}\right)
$$

and so

$$
\frac{a_{i}^{(k)}-a}{p^{k-1}} \equiv \frac{a_{i^{\prime}}^{\left(k^{\prime}\right)}-a}{p^{k-1}}(\bmod p)
$$

The left-hand side here is congruent to $i$ and the right to 0 if $k^{\prime}$ exceeds $k$ and to $i^{\prime}$ if $k^{\prime}$ equals $k$. The first alternative is impossible since $i$ belongs to the reduced residue system modulo $p$, and the second implies that the two AP's are identical.

Similarly $\left\langle a_{i}^{(k)}, d_{i}^{(k)}\right\rangle$ intersecting $\langle a, d\rangle$ would imply

$$
a_{i}^{(k)} \equiv a\left(\bmod p^{k}\right)
$$

and thus

$$
\frac{a_{i}^{(k)}-a}{p^{k-1}} \equiv 0(\bmod p)
$$

This is a contradiction since the left is congruent to $i$ modulo $p$.

Corollary 1. With $\mathscr{A}$ as in the theorem, let $n$ and $\beta$ be integers satisfying

$$
0 \leqslant n \leqslant p^{\alpha}, \quad 0<\beta \leqslant \alpha
$$

and

$$
\mathscr{B}=\bigcup_{s=1}^{n}\left\langle b_{s}, p^{\alpha}\right\rangle
$$

where the numbers $b_{s}$ are distinct modulo $p^{\alpha}$. Then

$$
\left|\left\{\langle a, d\rangle \in \mathscr{A}: p^{\beta} \mid d,\langle a, d\rangle \cap \mathscr{B}=\phi\right\}\right| \geqslant(\alpha-\beta+1)(p-1)+1-n .
$$

Proof. By the theorem, $\mathscr{A}$ contains the $(p-1)(\alpha-\beta+1)+1$ AP's

$$
\left\langle a_{i}^{(k)}, d_{i}^{(k)}\right\rangle \quad \text { for } \quad k=\beta, \ldots, \alpha, i=1, \ldots, p-1
$$

and

$$
\langle a, d\rangle \text {. }
$$

Each of these has modulus divisible by $p^{\beta}$. Now suppose both $\left\langle a_{i}^{(k)}, d_{i}^{(k)}\right\rangle$ and $\left\langle a_{i^{\prime}}^{\left(k^{\prime}\right)}, d_{i^{\prime}}^{\left(k^{\prime}\right)}\right\rangle$ intersect $\left\langle b_{s}, p^{\alpha}\right\rangle$ and that $k^{\prime} \geqslant k$. Then by (ii) of Lemma 1 ,

$$
a_{i}^{(k)} \equiv a_{i^{\prime}}^{\left(k^{\prime}\right)}\left(\bmod p^{k}\right)
$$

which leads to a contradiction as in part (ii) of the theorem. Similarly no $\left\langle a_{i}^{(k)}, d_{i}^{(k)}\right\rangle$ will intersect $\left\langle a, p^{\alpha}\right\rangle$, which contains $\langle a, d\rangle$. Thus at most $n$ of our AP's will intersect AP's in 0 leaving at least $(\alpha-\beta+1)(p-1)+1-n$ nonintersecting AP's.

\section{The second theorem.}

THEOREM 2. If $\mathscr{A}$ is regular, $P$ is the least common multiple of the moduli of the $A P$ 's in $\mathscr{A}, D$ divides $P$ and $D$ does not equal $P$, then

$$
|\{\langle a, d\rangle \in \mathscr{A}: d \nmid D\}| \geqslant 1+f\left(\frac{P}{D}\right) .
$$

Proof. We prove the theorem by induction on $v(P)$, the number of distinct prime divisors of $P$.

If $v(P)$ equals 1 then

$$
P=p^{\alpha}, \quad D=p^{\beta}, \quad 0 \leqslant \beta<\alpha,
$$

where $p$ is a prime. We then have

$$
\left|\left\{\langle a, d\rangle \in \mathscr{A}: d \chi p^{\beta}\right\}\right|=\left|\left\{\langle a, d\rangle \in \mathscr{A}: p^{\beta+1} \mid d\right\}\right| .
$$

By Corollary 1 this is not less than

$$
(\alpha-(\beta+1)+1)(p-1)+1=f\left(p^{\alpha} / p^{\beta}\right)+1
$$

This shows that the theorem holds when $v(P)$ equals 1 .

To continue the induction suppose that the theorem holds for $v(P)$ not exceeding $n$. Let $\mathscr{A}$ be regular and let the least common multiple of the moduli of the AP's in $\mathscr{A}$ be $p^{\alpha} P$, where $p$ is a prime not dividing $P$ and $v(P)$ 
equals $n$, so that $v\left(p^{\alpha} P\right)=n+1$. We will write the AP's in $s$ in the form $\left\langle a, p^{y} d\right\rangle$ where $p$ does not divide $d$. We must find a lower bound for

$$
\left|\left\{\left\langle a, p^{\gamma} d\right\rangle \in, \alpha: p^{y} d \nmid p^{\beta} D\right\}\right|
$$

where $p$ does not divide $D$.

We now introduce some notation. For each residue class $s$ modulo $p^{\alpha}$ let $\mathscr{N}_{s}$ be a minimal subcollection of $\&$ that covers $\left\langle s, p^{\alpha}\right\rangle$. It is clear that such a subcollection exists. We then set

$$
P_{s}=\operatorname{lcm}\left\{d:\left\langle a, p^{\gamma} d\right\rangle \in \mathbb{A}_{s}\right\},
$$$$
R_{0}=D \text {, }
$$

$R_{s}=\operatorname{lcm}\left\{R_{s-1}, P_{s}\right\}$,

$D_{s}=\left(R_{s-1}, P_{s}\right)$,

$Q_{s}=\left\{\left\langle a, p^{\gamma} d\right\rangle \in s_{s}: d \nmid D_{s}\right\}$, for $s=1, \ldots, p^{\alpha}$.

We remark that:

$$
\begin{gathered}
\frac{P_{s}}{D_{s}}=\frac{R_{s}}{R_{s-1}} \quad \text { for } \quad s=1, \ldots, p^{\alpha}, \\
R_{p^{\alpha}}=P,
\end{gathered}
$$$$
Q_{s} \text { is empty if } D_{s}=P_{s} \text {, }
$$

$$
Q_{s}=\left\{\left\langle a, p^{z} d\right\rangle \in \alpha_{s}: d \mid R_{s}, d \nmid R_{s-1}\right\} \text {. }
$$

It is clear from the last remark and from the definition of $R_{s}$ that the collections $Q_{s}$ are pairwise disjoint.

Claim. If $D_{s}$ does not equal $P_{s}$,

$$
\left|Q_{s}\right| \geqslant f\left(\frac{P_{s}}{D_{s}}\right)+1
$$

Proof of Claim. Since $\mathscr{L}_{s}$ is a minimal covering of $\left\langle s, p^{\alpha}\right\rangle$ we may reduce it to get a regular covering $\mathscr{N}_{s}^{*}$. Any $\mathrm{AP}\left\langle a, p^{\gamma} d\right\rangle$ in $\mathscr{A}_{s}$ will be reduced, according to Lemma 3 , to an AP of the form $\left\langle a^{*}, d\right\rangle$. Since $D_{s} \mid P_{s}$ and $v\left(P_{s}\right)$ is at most $n$, it follows from the induction hypothesis that if $D_{s}$ does not equal $P_{s}$,

$$
\left|Q_{s}\right|=\left|\left\{\left\langle a^{*}, d\right\rangle \in \ldots \mathscr{O}_{s}^{*}: d \nmid D_{s}\right\}\right| \geqslant f^{\prime}\left(\frac{P_{s}}{D_{s}}\right)+1 \ldots
$$

We now obtain a lower bound for the cardinality of the set $\left\{\left\langle a, p^{\gamma} d\right\rangle \in \mathscr{A} A: p^{y} d \nmid p^{\beta} D\right\}$. We note that,

$$
p^{y} d \chi p^{\beta} D \Rightarrow p^{\beta+1} \mid p^{y} \text { or } d \chi D \text { and } \bigcup_{s=1}^{\alpha} \mathscr{A} \mathscr{H}_{s}=\mathscr{A}
$$

Therefore the cardinality equals

$$
\left|\left(\bigcup_{s=1}^{p^{\alpha}}\left\{\left\langle a, p^{\gamma} d\right\rangle \in \mathscr{A _ { s }}: d \nmid D\right\}\right) \cup\left\{\left\langle a, p^{\gamma} d\right\rangle \in \mathscr{A}: p^{\beta+1} \mid p^{\gamma}\right\}\right| .
$$

Each collection in the first union contains a subcollection

$$
\left\langle\left\langle a, p^{\gamma} d\right\rangle \in \mathscr{A}_{s}: d \nmid D_{s}\right\}=Q_{s}
$$

so the required cardinality is at least

$$
\begin{aligned}
& \left|\left(\bigcup_{s=1}^{p^{\alpha}} Q_{s}\right) \cup\left\{\left\langle a, p^{y} d\right\rangle \in \cdot d: p^{\beta+1} \mid p^{y}\right\}\right| \\
& \geqslant \sum_{\substack{s=1 \\
P_{s} \neq D_{s}}}^{p^{\alpha}}\left|Q_{s}\right|+\left|\left\{\left\langle a, p^{\gamma} d\right\rangle \in \mathscr{S} \backslash \bigcup_{\substack{s=1 \\
p_{s} \neq D_{s}}}^{p^{\alpha}} \mathscr{S}_{s}: p^{\beta+1} \mid p^{\gamma}\right\}\right| .
\end{aligned}
$$

By (4.1) to (4.5) and the additivity of $f$,

$$
\begin{aligned}
\sum_{\substack{s=1 \\
P_{s} \neq D_{s}}}^{p^{\alpha}}\left|Q_{s}\right| & \geqslant \sum_{\substack{s=1 \\
P_{s} \neq D_{s}}}^{p^{\alpha}} f\left(\frac{P_{s}}{D_{s}}\right)+\sum_{\substack{s s=1 \\
P_{s} \neq D_{s}}}^{p^{\alpha}} 1=\sum_{s=1}^{p^{\alpha}} f\left(\frac{R_{s}}{R_{s-1}}\right)+\sum_{\substack{s=1 \\
P_{s} \neq D_{s}}}^{p^{\alpha}} 1 \\
& =f\left(\frac{P}{D}\right)+\sum_{\substack{s=1 \\
P_{s} \neq D_{s}}}^{p^{\alpha}} 1 .
\end{aligned}
$$

We now consider the second term in (4.6). We put

$$
B=\bigcup_{\substack{s=1 \\ p_{s} \neq p_{s}}}^{p^{\alpha}}\left\langle s, p^{\alpha}\right\rangle
$$

and note that if the intersection of $\left\langle a, p^{\gamma} d\right\rangle$ and $\left\langle s, p^{\alpha}\right\rangle$ is empty then $\left\langle a, p^{y} d\right\rangle$ does not belong to $\mathscr{A}_{s}$, so the second term in (4.6) is at least

$$
\left\{\left\{\left\langle a, p^{\gamma} d\right\rangle \in \mathscr{A}:\left\langle a, p^{\gamma} d\right\rangle \cap B=\varnothing, p^{\beta+1} \mid p^{\gamma}\right\} \mid\right. \text {. }
$$

By Corollary 1 this is at least

$$
(\alpha-(\beta+1)+1)(p-1)+1-\sum_{\substack{s=1 \\ p_{s} \neq D_{s}}}^{p^{\alpha}} 1=f\left(\frac{p^{\alpha}}{p^{\beta}}\right)+1-\sum_{\substack{s=1 \\ P_{s} \neq D_{s}}}^{p^{\alpha}} 1 .
$$

On adding the right-hand sides of (4.7) and (4.8) we obtain the required lower bound. That is,

$$
\begin{aligned}
& \left\{\left\langle\left\langle a, p^{\gamma} d\right\rangle \in \mathscr{A}: p^{\gamma} d \nmid p^{\beta} D\right\}\right.
\end{aligned}
$$

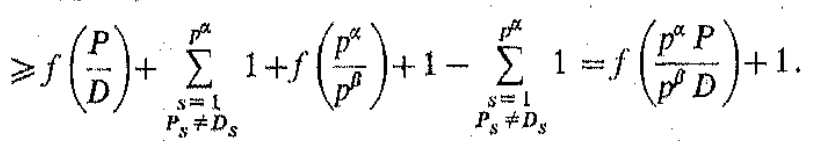

Thus the theorem holds when the least common multiple of the moduli has $n+1$ distinct prime factors and the theorem is proven by induction.

Corollary 2. If $\mathscr{A}$ is regular, $P$ the least common multiple of the moduli of the $A P^{\prime}$ 's in $\mathscr{A}$ then

$$
\mid \cdot \mathscr{A} \geqslant f(P)+1
$$


Proof. If $P$ does not equal 1 the result is immediate on putting $D$ equal 1 in the theorem. If $P$ equals 1 then $\mathscr{A}$ must be $\{\langle 0,1\rangle\}$ and the result still holds.

Now let $d$ be a collection of AP's, not necessarily regular, which covers the integers. Znám ([5]) defines an AP $\left\langle a_{0}, d_{0}\right\rangle$ in $\mathscr{A}$ as essential if $\mathscr{A} \backslash\left\langle a_{0}, d_{0}\right\rangle$ does not cover the integers. The following result extends Theorem 1 of [5].

COROllary 3. If $\mathscr{A}$ covers the integers, $\left\langle a_{0}, d_{0}\right\rangle$ is essential in $\mathscr{N}$, then

$$
\left|\left\{\langle a, d\rangle \in \mathscr{A}:\left(d, d_{0}\right)>1\right\}\right| \geqslant f\left(d_{0}\right)+1 \text {. }
$$

Proof. Let $\mathscr{A}^{*}$ be a regular subcollection of $\mathscr{A}$, and $P$ the least common multiple of the moduli of the AP's in it. It is clear that $\left\langle a_{0}, d_{0}\right\rangle$ belongs to $\mathscr{A}^{*}$ and hence that $d_{0}$ divides $P$. Then

$$
\left|\left\{\langle a, d\rangle \in \mathscr{A}:\left(d, d_{0}\right)>1\right\}\right| \geqslant\left|\left\{\langle a, d\rangle \in \mathscr{A}^{*}: d \times \frac{P}{d_{0}}\right\}\right| \geqslant f\left(d_{0}\right)+1 \text {. }
$$

Acknowledgement. The author wishes to thank Dr. J. Pitman for her unrelenting assistance in the preparation of this paper.

\section{References}

[1] I. Korec, On a generalization of Mycielski's and Znám's conjectures about coset decomposition of Abelian groups, Fund. Math. 85 (1974), pp. 41-48.

[2] S. Znám, On Mycielski's problem on systems of arithmetic sequences, Colloq. Math. 15 (1966), pp. 201-204.

[3] - A remark to a problem of J. Mycielski on arithmetic sequences, ibid. 20 (1969), pp. 69-70.

[4] - On exactly covering systems of arithmetic sequences, Colloquia Math. Soc. János Bolyai, 2. Number Theory, Debrecen 1968.

[5] - On properties of systems of arithmetic sequences, Acta Arith. 26 (1975), pp. 279-283.

THE UNIVERSITY OF ADELAIDE

G.P.O. Box 498, Adelaide

Received on 5.10 .1983

and in revised form on 20.3.1984

\section{Verzichtbare und unverzichtbare Elemente bei der Darstellung als Summe und als Differenz von Quadraten}

von

Erhard Deinert, Erich HArtter und Joachim Zollner (Mainz)

Wir bezeichnen die Menge der Quadrate der ganzen Zahlen mit $Q_{0}$. Nach einem bekannten Satz von Lagrange ist jede natürliche Zahl als Summe von vier Elementen aus $Q_{0}$ darstellbar. In [3] wurde gezeigt, da $\beta$ es unendliche Mengen $S \subset Q_{0}$ gibt, so daß jede natürliche Zahl auch noch als Summe von vier Quadraten aus $Q_{0} \backslash S$ darstellbar ist. Erdös und Nathanson [2] haben darüber hinaus die Existenz von Mengen $S$ mit dieser Eigenschaft und $\left|\left(Q_{0} \backslash S\right) \cap[0, x]\right| \leqslant C x^{3 / 8+\varepsilon}$ für beliebiges $\varepsilon>0$ nachgewiesen, wobei $C>0$ nur von $\varepsilon$ abhängt $\left({ }^{1}\right)$. In der vorliegenden Arbeit werden genau die Quadrate bestimmt, die in jedem Fall in $Q_{0} \backslash S$ noch enthalten sein muissen. Solche Quadrate nennen wir unverzichtbar.

Sei $Q$ die Menge der Quadrate der natürlichen Zahlen. Genau die von 1 und 4 verschiedenen naturlichen Zahlen, die $\not \equiv 2(\bmod 4)$ sind, lassen sich als Differenz zweier Quadrate aus $Q$ darstellen. In [3] wurde gezeigt, daß dies auch noch mit den Quadraten aus $Q \backslash T$ möglich ist, wobei $T \subset Q$ eine geeignete unendliche Menge von Quadraten ist. Auch in diesem Fall werden die unverzichtbaren Quadrate charakterisiert.

1. Zunächst führen wir einige Bezeichnungen ein $\left({ }^{2}\right)$ :

Sei $E\left(n^{2}\right)$ die Menge aller $z \in N_{0}$, für die gilt: Aus $z=a_{1}^{2}+a_{2}^{2}+a_{3}^{2}+a_{4}^{2}$ mit $a_{i} \in N_{0}(i=1,2,3,4)$ folgt $a_{i}^{2}=n^{2}$ für mindestens ein $i$.

Sei weiter $U:=\left\{n^{2} \mid E\left(n^{2}\right) \neq \varnothing\right\}$ und $\tilde{U}:=\left\{n^{2}|| E\left(n^{2}\right) \mid=\infty\right\}$.

Sei analog $E^{-}\left(n^{2}\right)$ die Menge aller $z \in N$, fur die gilt: Aus $z=a_{1}^{2}-a_{2}^{2}$ mit $a_{i} \in N(i=1,2)$ folgt $a_{i}^{2}=n^{2}$ fur $i=1$ oder für $i=2$.

Entsprechend wie oben sei dann $U^{-}:=\left\{n^{2} \mid E^{-}\left(n^{2}\right) \neq \varnothing\right\}$ und $\tilde{O}^{-}$ $:=\left\{n^{2}|| E^{-}\left(n^{2}\right) \mid=\infty\right\}$.

(1). Nathanson [5] zeigte, daß sogar $\left|\left(Q_{0} \backslash S\right) \cap[0, x]\right| \leqslant C \dot{x}^{1 / 3+z}$ gilt.

(2) $N$ ist die Menge der natürlichen Zahlen; $N_{0}=N \cup\{0\}$ die Menge der nichtnegativen ganzen Zahlen. 